\title{
Correction of WOW and Flutter in Audio Signals using Least Mean Square Algorithm
}

\author{
Chanda Thakur \\ Research Scholar \\ ECE Dept. \\ SUSCET, Tangori
}

\author{
Satbir Singh \\ Assistant Professor \\ ECE Dept. \\ SUSCET, Tangori
}

\begin{abstract}
This paper describes a system which is used to remove Wow and Flutter from audio signal using Least Mean Square Algorithm. It is impossible to completely remove Wow and Flutter from audio signal but its effect can be reduced significantly. It occurs during the process of sound reproduction. It is the group of tones created by the irregularities in turntables or tape drive speed during reproduction, duplication or recording. Wow occurs due to irregularities at low frequency whereas at high frequency irregularities, Flutter occurs. Least Mean Square Algorithm uses Adaptive Filter which adjusts their coefficient in order to minimize the required wobble effects in audio signal. Results show that LMS has comprehensively diminished the effects of wow and flutter.
\end{abstract}

\section{Keywords}

Wow and Flutter, Adaptive Filtering, Least Mean Square Algorithm

\section{INTRODUCTION}

Wow and Flutter is the distortion of audio signal. Basically Wow occur at the range of $0.5 \mathrm{~Hz}$ to $6 \mathrm{~Hz}$ so Wow is called as low frequency distortion of audio signal whereas Flutter occur at the range of $6 \mathrm{~Hz}$ to $100 \mathrm{~Hz}$ so Flutter it called as high frequency distortion of audio signal [1]. Wobble is caused by the speed fluctuation in tape. Both Wow and Flutter are separate terms but we combined them so that we can measure them in a same account simultaneously. It is found that Flutter is at its peak value when actual frequency of wobble is $4 \mathrm{kHz}$ and its value is least at below and above of the frequency 4 $\mathrm{kHz}$. This is clearly demonstrated by the following diagram. [2]

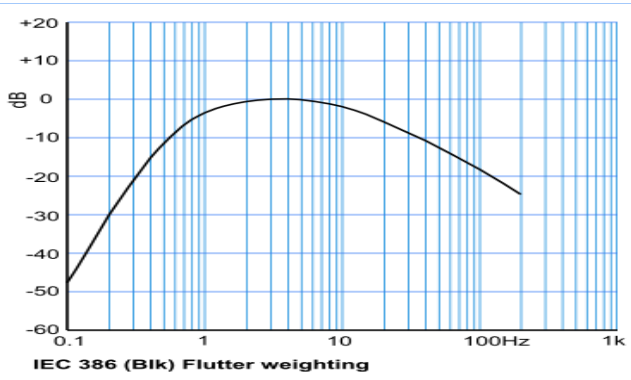

Fig 1: Variation of wow and flutter

Effect of Flutter and Wow is listened when music is played on guitar, piano, flute, etc. Wow creates variation in pitch while Flutter makes the sound of music differently, making the sound ugly or cracked. The reason behind that is when a signal is recorded with least amount of Flutter approximate $0.1 \%$ then human being can listen that the sound of signal is fine in dead room but in a reverberant room they can heard fluctuation of sound signal clearly. Another form of flutter is known as scrape flutter or high frequency flutter whose range is above $100 \mathrm{~Hz}$. Scrape flutter causes vibrations in tape when it passes over a head. As a result of this there is rapidly interacting stretching occur in the tape and at the head. This condition is known as 'scrape flutter'. Scrape flutter adds a roughness to the sound. It is not typical like wow \& flutter, and damping devices or heavy rollers sometimes it put to use on professional tape machines to prevent it. To measure the Scrape flutter special techniques are required, often using a $10 \mathrm{kHz}$ tone. To correct wow and flutter, novel DSP processes have been developed. It tracks various factors on the tape or film which can be up cycle as timing references. In 2006, some DVD releases that utilized a system which is developed by Plangent Processes [2]. It reduces the effect of wow and flutter from very high rates to extremely low levels, with actual improvement in quality, and without adding any distortion or extra cycles of sound. To eliminate wow and flutter software named as capstan has been invented by Celemony [3]. It analyzes the musical material which is already in digitized form. Capstan is the latest method to control the effects of wow and flutter problems. It detects the problem of pitch in the audio signal and corrects this problem by increasing or decreasing the speed of playback of audio file. The present research work involves reduction of Wow and Flutter using the LMS algorithm. This algorithm is easy to implement in software as well as hardware because of its computational simplicity. The LMS algorithm had already been used in various applications encompassing speech and image processing including signal interference, channel equalization, time varying system identification [4], image denoising and image compression, etc. The section 1 provides introductory part; section 2 explains the LMS algorithm in detail along with explaining the major causes of wow and flutter respectively. Section 3 describes the methodology used for the project. Results and Discussion are presented in section 4 and section 5 concludes the paper.

\section{LEAST MEAN SQUARE ALGORITHM}

LMS stands for Least Mean Square. This algorithm was developed by Widrow and Hoff in 1959 through their studies in pattern recognition [5]. Since its invention, it has been in use for variety of signal processing applications including denoising of image. Since images are suffered from white Gaussian noise so, least mean square algorithm is used to denoise the images. It is also implemented in image compression which is very demanding application in telecommunication image compression. This technique is used to control the distortion in image after transmitting the image through channel. In the presence of numerical errors caused by finite precision arithmetic LMS performs robustly. There is efficient use of memory. It does not require prior knowledge of signal statics. In least mean square algorithm weights are obtained on the basis of estimation but their value improves constantly with time as weights are adjusted [6].Its 
convergence rate is not too express because of eigen value spread. It is stochastic gradient descent method in which adaptive filter is used to minimize the error [7]. At each iteration, to make the exact approximations, weight vector is computed by steep descent algorithm and it converges to the optimum wiener solution. The exact measurement of gradient vector is impossible because it would require prior knowledge of both the matrix that are tap input autocorrelation matrix $\mathrm{R}$ and the cross correlation vector $\mathrm{p}$. The gradient vector must be estimated from the given data when somebody is doing work in unknown environment. After the estimation of gradient vector a relation is obtained through which it can update the tap weight vector by using the following equations:

$\mathrm{w}(\mathrm{n}+1)=\mathrm{w}(\mathrm{n})+\mu \mathrm{u}(\mathrm{n})\left[\mathrm{d}^{*}(\mathrm{n})-u^{H}(\mathrm{n}) \mathrm{w}(\mathrm{n})\right.$

where, $\mu=$ step size parameter

$u^{H}(\mathrm{n})=$ Hermit of a matrix $\mathrm{u}$

$d^{*}(n)=$ Complex conjugate of $d(n)$

The above mentioned equation may write the result in the form of three basic relations as follows:

1. Filter output

$$
y(n)=w^{H}(n) u(n)
$$

2. Estimation Error or Error Signal

$$
e(n)=d(n)-y(n)
$$

3. Tap weight adaptation:

$$
W(n)=w(n)+\mu u(n) e *(n)
$$

From equation (2) and (3) the error $e(n)$ can be calculated and its calculation is based on the current tap weight vector $w(n)$. In equation (4) the term $u(n) e *(n)$ represents an adjustment to calculate the current tap weight vector $w(n)$. Iteration process is started from the initial value that is $w(0)$. At each iteration algorithm need to know three recently used values and these are $w(n), \mathrm{u}(\mathrm{n})$ and $d(n)$.

\subsection{Least mean square algorithm using adaptive filtration}

Adaptive filter is a self adjusted filter. The term self adjusted is used because its coefficients are not fixed, it reduce the error in the original signal by adjusting its coefficients. Transversal filter is the most common form of adaptive filter so, we use least mean square algorithm [5]. By using the concept of adaptive filtrations LMS has been implemented in equalization of data communication channel [8]. Data can be transmitted reliably through a communication channel and demodulate the signal perfectly at the output without causing any error if the channel is ideal. It is used in time varying system identification [4]. System identification is the central issue that occurs in various applications of least mean square algorithm such as in echo cancellation, channel equalization and teleconferencing etc. To find the parameter of unknown model the input- output desired response can be calculated and error that is optimized.

Basic concept of adaptive filter is more clear by the given below diagram of adaptive filter.

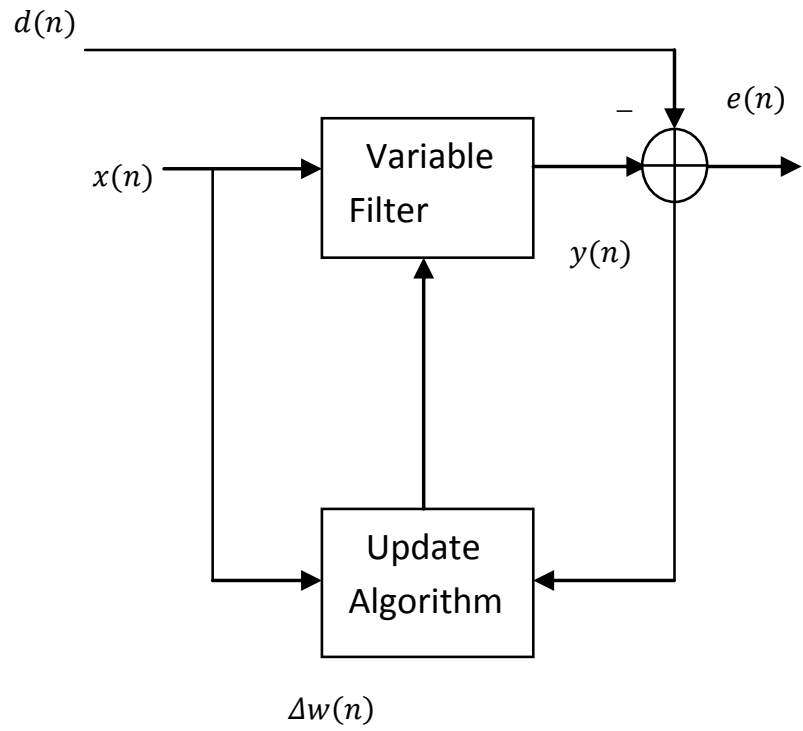

Fig 2: Adaptive Filter

\subsection{Wow and Flutter on the basis of their causes}

Following are some of causes of wow and flutter:

Tape Speed: Speed of tape is controlled by motor and the pinch roller. Pressure must be maintained within specification since a high take up tension will pull the tape between the motor and pinch roller. Due to this motor is not able to control the speed of tape. Similarly, if a high back tension is present then motor cannot also control the speed of tape. Since the capstan motor is synchronous in nature then it leads to variation in tape speed if main frequency is not correct. These variations results in fluctuation and this become the cause of wow and flutter in audio signal.

Wrong Tape Tension: Wrong tape tension introduces error when tape is dragged at different standard value of tension. These error results in wow and flutter so, tape tension is important to control in all modes of tape machines.

1. During playback: A certain amount of tape tension is required during playback mode in order to provide a good head contact with tape.

2. During spooling: A certain amount of tape tension is required during spooling mode in order to provide a safe running of tape.

3. During spooling and breaking: A certain amount of tape tension is required during spooling and breaking in order to prevent from over stressing and stretching of tape.

Other Reasons: If moist and sticky tape, wrong break adjustment, wrong pinch roller pressure, dirty capstan, dirty pinch roller, bearing problems and worn out idler, etc.

\section{METHODOLOGY}

In the current work, we have taken a sample of audio signal which is suffered by wow and flutter. This signal act as input signal which is represented by d (n). Another signal x (n) has also been taken that is in the frequency range of 0.5 to $100 \mathrm{~Hz}$ and acts as a reference input. LMS algorithm subtracts the input signal from the signal received from the filter output and produces the required updating of weights using which a new 
recovered signal is produced which is liberated from wow and flutter. The proposed block diagram is explained in fig.3:

\section{EXPERIMENTAL RESULTS}

The digital signal samples of the converted analog recorded tapes were taken [3] and the results obtained using LMS algorithm are shown in this section. Since using LMS, we have following two parameters in hand to train the filter adaptively:

1. Learning step parameter

2. Reference input signal

So, the above two parameters can be varied in order to minimize the effects of wow and flutter.
SIGNAL 1: For signal 1[3], a wave/mp3 file, the following waveforms are obtained for $\mu=0.008$ and a reference signal of $43 \mathrm{kHz}$ and length of the signal is 751104 samples.

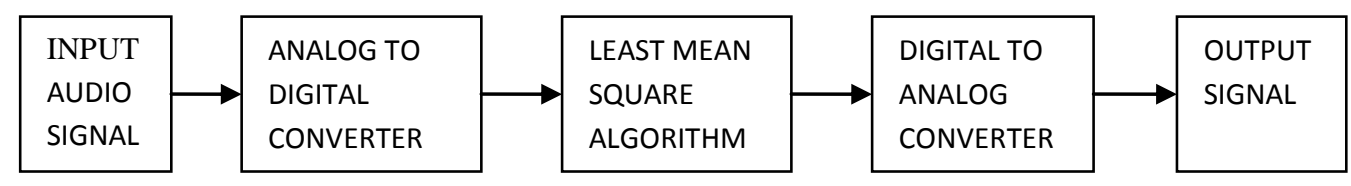

Fig 3: Correction of wow and fluttered audio signal by using least mean Square algorithm

Input Signal: Input signal $d(n)$ contain the audio signal which is affected by wow and flutter. Variation occurs in the speed of playback recorded signal causes variation and distortion in pitch. These variations and distortions result in fluctuation of signal. Due to fluctuations low frequency irregularities and high frequency irregularities occurs which produces wow and flutter. These signals are taken from analog tape recorders and gramophones.

Analog to digital converter: Analog to digital converter is a device which converts analog signals into digital signals. Analog to Digital converter is used so that computer can understand and process the coming wow ant flutter signal. The accuracy of converter to convert the audio signal into digital one depends on the sampling rate. Higher the sampling rates mean higher accuracy of conversion. Thus computer digital signal can now be processed using any computer algorithm.

Least mean square algorithm: The digital signal thus obtained from above step is now fed as a input to Least Mean Square algorithm. LMS adjusts the weight in such a way that it minimizes the irregularities in signal. It also minimizes the effect of wow and flutter in digital signal.

Digital to analog converter: Digital to analog converter device converts the digital signal back to analog form. This signal gets relaxed from the effects of audio wobbles and hence can be heard accurately.

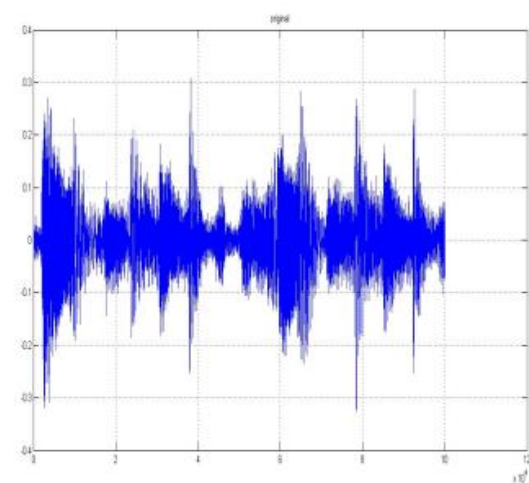

(a)

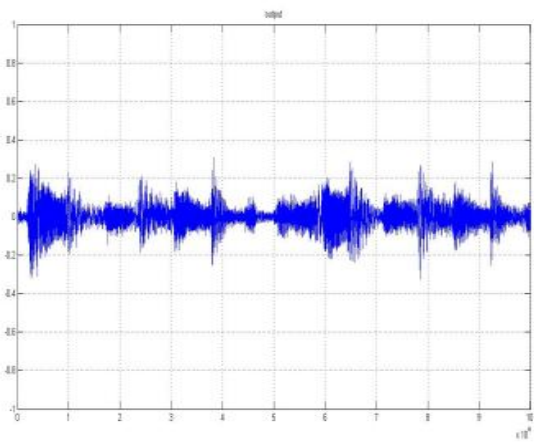

(b) 


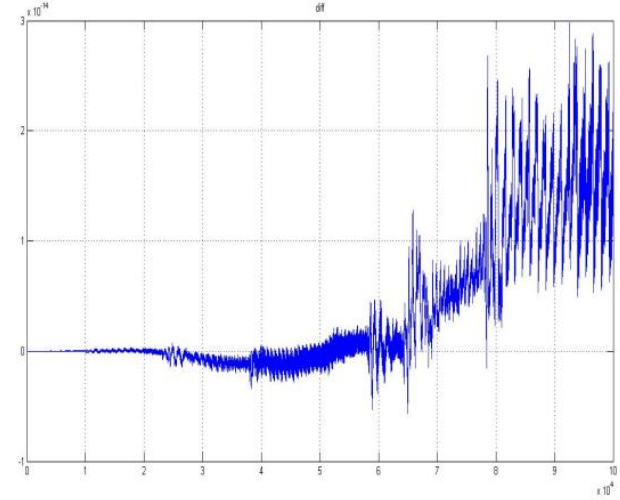

(c)

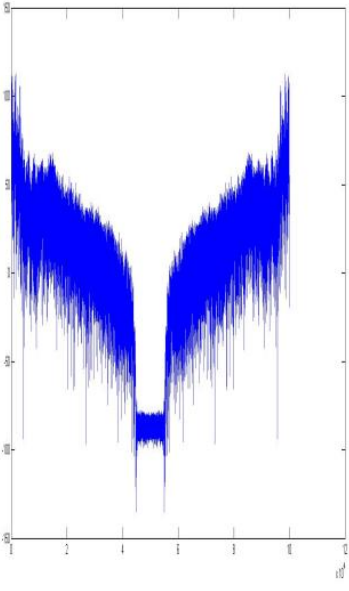

(d)

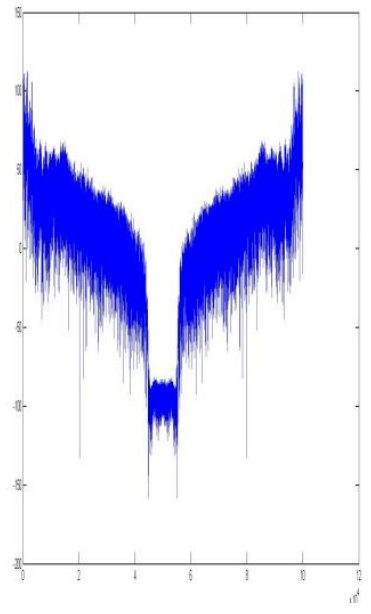

(e)
Fig 4: (a) Original Input signal containing wow and flutter (b) Recovered corrected output signal (c) Difference between original and corrected signal (d) Spectrum of original signal (e) Spectrum of recovered signal.

SIGNAL 2: For signal 2[3], a wave/mp3 file, the following waveforms are obtained for $\boldsymbol{\mu}=\mathbf{0 . 1}$ and a reference signal of $43 \mathrm{kHz}$ and length of the signal is 751104 samples.

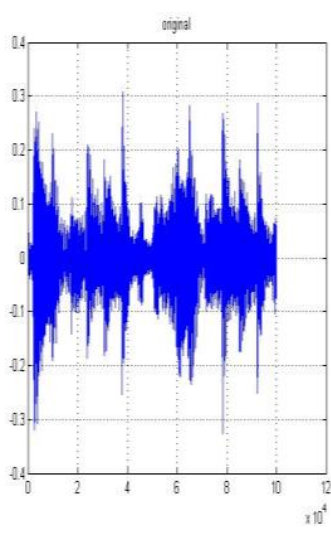

(a)

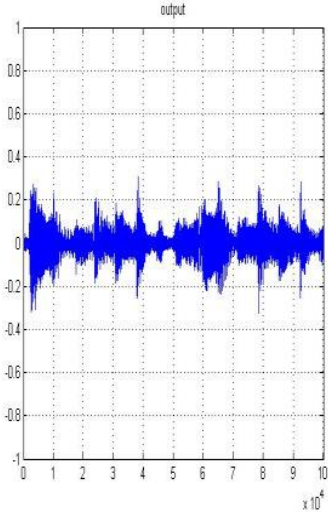

(b)

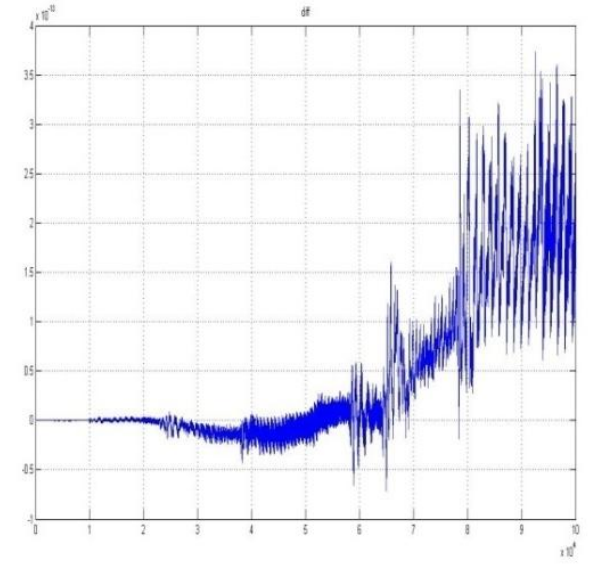

(c)

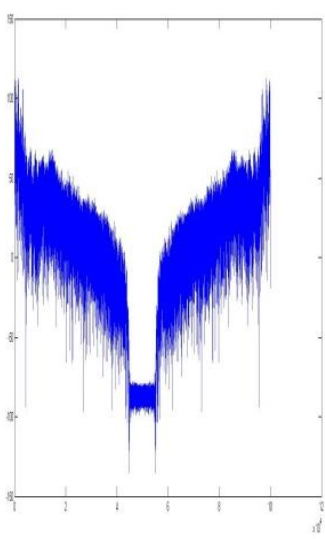

(d)

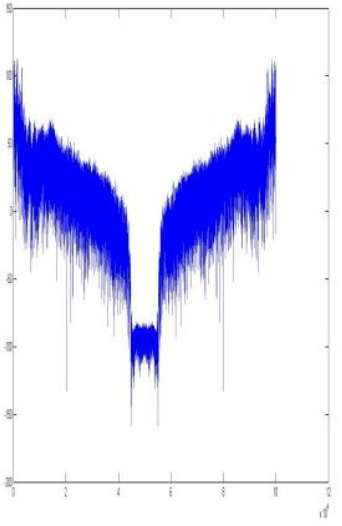

(e)
Fig 5: (a) Original Input signal containing wow and flutter (b) Recovered corrected output signal (c) Difference between original and corrected signal (d) Spectrum of original signal (e) Spectrum of recovered signal

SIGNAL 3: For signal 3[3], a wave/mp3 file, the following waveforms are obtained for $\boldsymbol{\mu}=\mathbf{0 . 8}$ and a reference signal of $43 \mathrm{kHz}$ and length of the signal is 751104 samples.

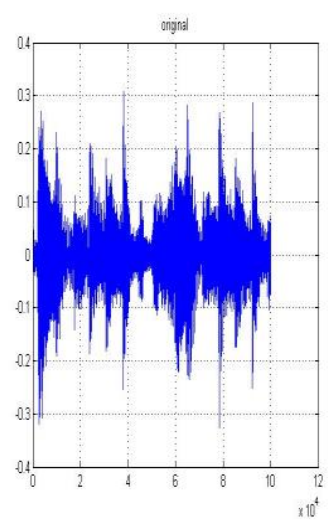

(a)

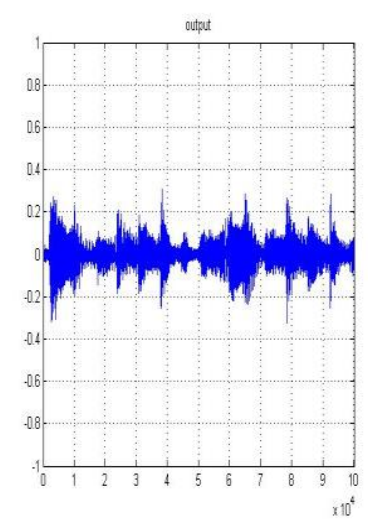

(b) 


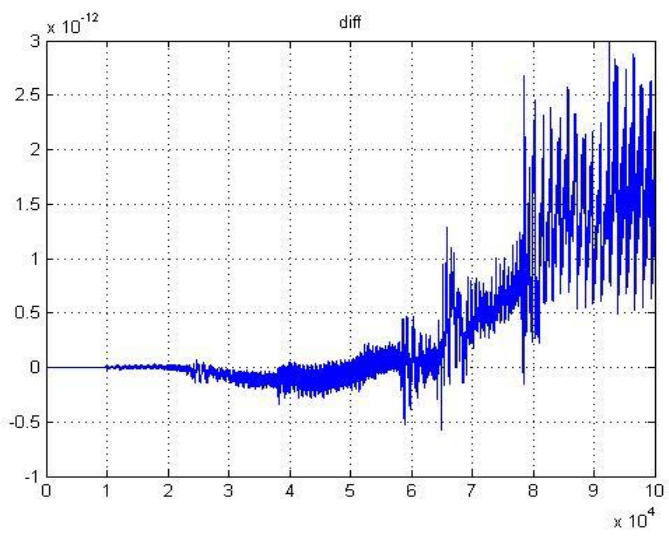

(c)

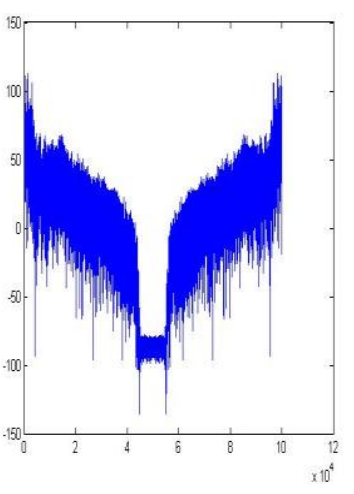

(d)

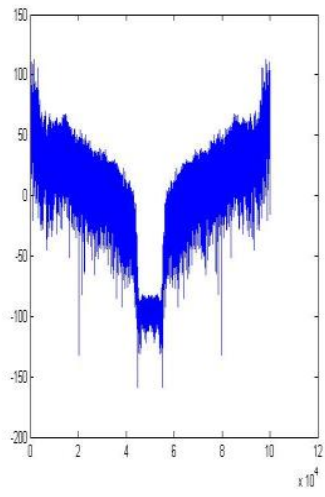

(e)

Fig 6: (a) Original Input signal containing wow and flutter (b) Recovered corrected output signal (c) Difference between original and corrected signal (d) Spectrum of original signal (e) Spectrum of recovered signal

The reference signal taken as a input to the Adaptive filter based LMS lock is arbitrarily varied by keeping in mind the range of wow and flutter disturbances which lies between 3 $\mathrm{KHz}$ to $100 \mathrm{KHz}$. Another set of results are obtained by varying the reference signal to $3150 \mathrm{~Hz}$. And resulting waveforms are obtained below:

SIGNAL 4: For signal 4[3], a wave/mp3 file, the following waveforms are obtained for $\mu=0.008$ and a reference signal of $3150 \mathrm{~Hz}$ and length of the signal is 14512100 :

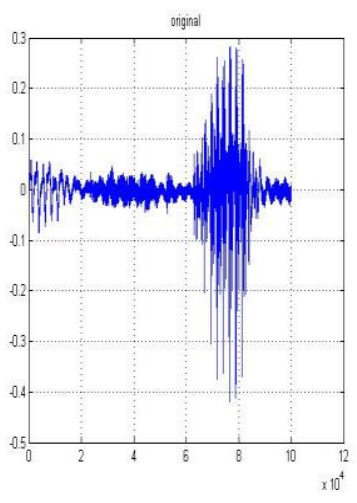

(a)

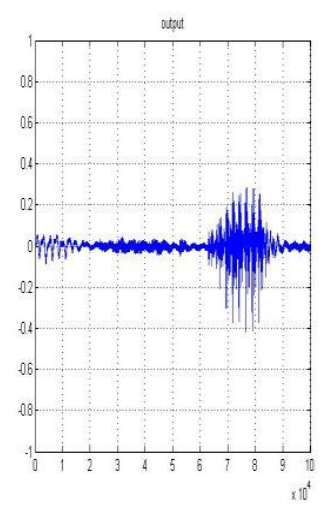

(b)

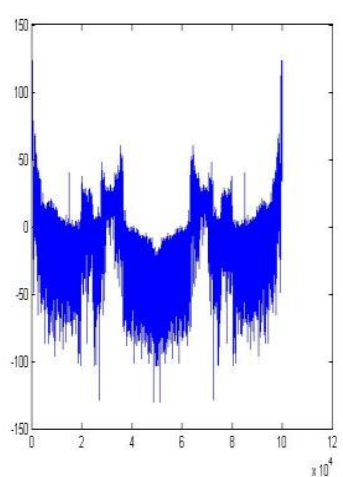

(d)

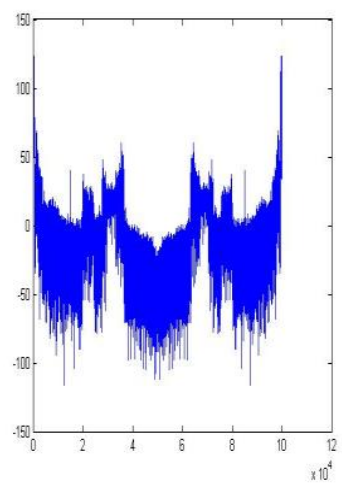

(e)

Fig 7: (a) Original Input signal containing wow and flutter (b) Recovered corrected output signal (c) Difference between original and corrected signal (d) Spectrum of original signal (e) Spectrum of recovered signal

SIGNAL 5: For signal 5[3], a wave/mp3 file, the following waveforms are obtained for $\mu=0.1$ and a reference signal of $3150 \mathrm{~Hz}$ and length of the signal is 14512100 :

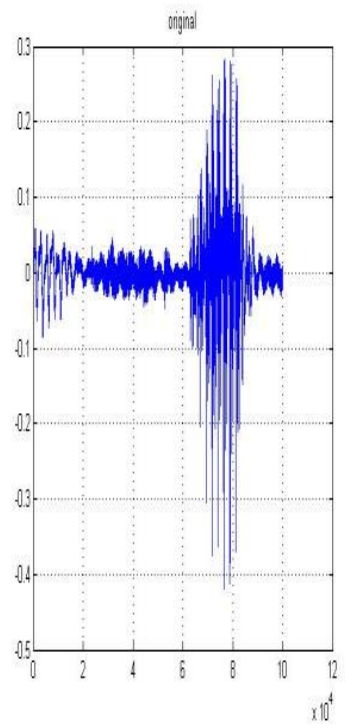

(a)

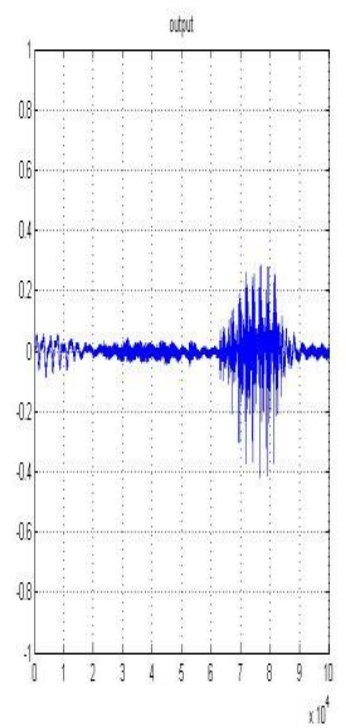

(b) 


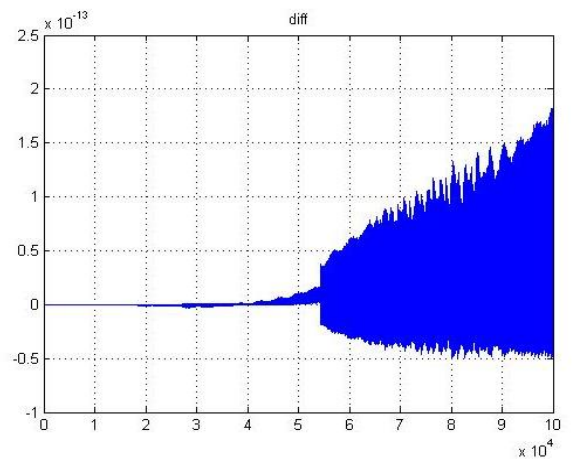

(c)

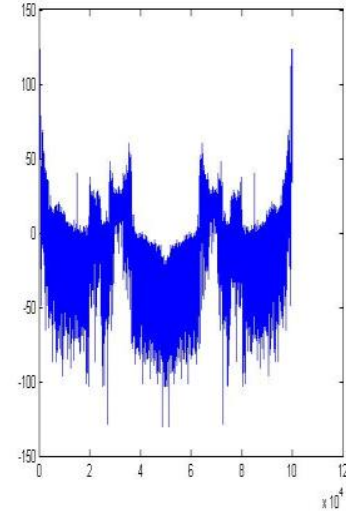

(d)

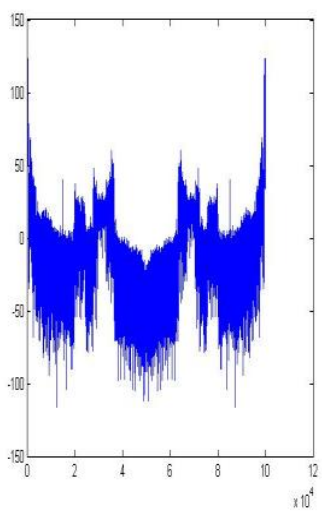

(e)
Fig 8: (a) Original Input signal containing wow and flutter (b) Recovered corrected output signal (c) Difference between original and corrected signal (d) Spectrum of original signal (e) Spectrum of recovered signal

SIGNAL 6: For signal 6[3], a wave/mp3 file, the following waveforms are obtained for $\mu=0.8$ and a reference signal of $3150 \mathrm{~Hz}$ and length of the signal is 14512100 :

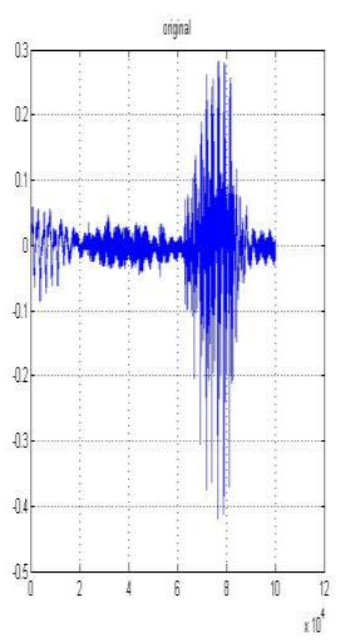

(a)

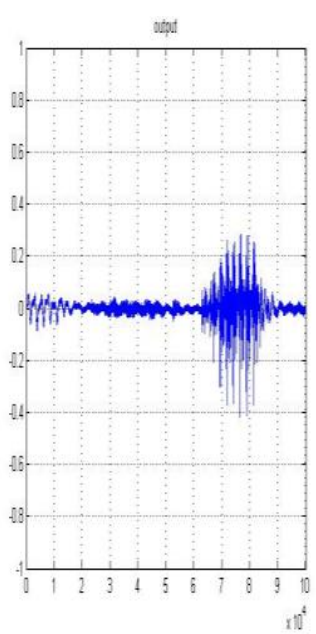

(b)

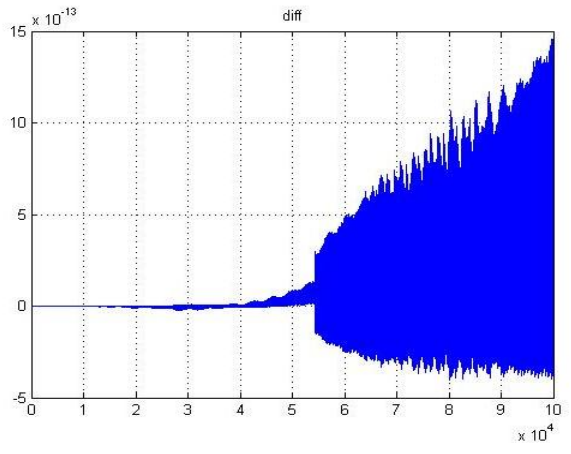

(c)

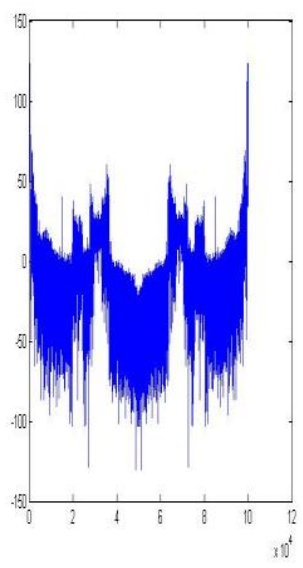

(d)

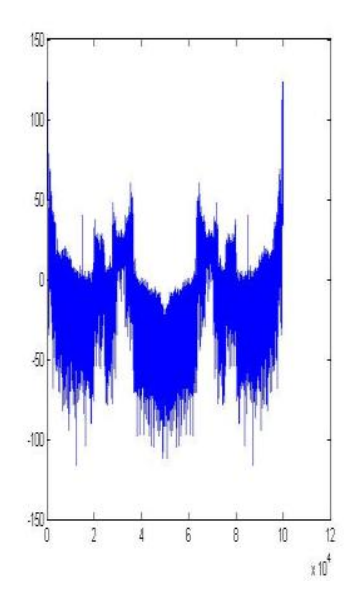

(e)
Fig 9: (a) Original Input signal containing wow and flutter (b) Recovered corrected output signal (c) Difference between original and corrected signal (d) Spectrum of original signal (e) Spectrum of recovered signal

\section{CONCLUSION}

In the above presented research work, it was observed that by the use of Least Mean Square algorithm, effects of wow and flutter present in audio signal, can be reduced significantly. The main deciding parameters are learning parameter $\mu$ and reference signal and by varying these for different values, we can obtain different outputs corresponding to adaptive learning of filter. The reference signal in LMS was chosen close to the frequency range of wow and flutter frequencies. This is the unique idea for minimizing the effect of wow and flutter with minimal work. In future, work can be done to reducing the effect of wow and flutter by applying other similar kind of algorithms such as Normalized Least Mean Square Algorithm, Variable Step Size Least Mean Square Algorithm, etc. which may add an improvement to the reduction of wobbles in audio signals.

\section{REFERENCES}

[1] P. MAZIEWSKI, "Evaluation of maximal modulation frequency for Wow and Flutter determination", Multimedia Systems Department, Gdansk University of Technology ul. Narutowicza 11/12, 80-952 Gdansk, Poland.

[2] http://www.lindos.co.uk

[3] http://www.plangentprocesses.com 
[4] Irina DORNEAN, Marina TOPA, Botond Sandor KIREI, Erwin SZOPOS, "System identification with least mean square adaptive algorithm", "Interdisciplinarity in Engineering"Scientific International conference, tg. mureş - românia, 15 -16 november 2007

[5] Jashvir Chhikara and Jagbir Singh, "Noise cancellation using Adaptive algorithm", International Journal of Modern Engineering Research (IJMER) www.ijmer.com Vol.2, Issue.3, May-June 2012 pp-792-795

[6] Sujith Chatrad \& Asha K S, "Area and Power Efficient LMS Algorithm for "Denosing Speech Signal", International Journal of Engineering Science and
Innovative Technology (IJESIT)Volume 2, Issue 4, July 2013.

[7] Radhika Chinaboina, D.S.Ramkiran, HabibullaKhan, M.Usha, B.T.P.Madhav, K.Phani Srinivas \& G.V.Ganesh, "Adaptive algorithm for acoustic echo cancellation in speech processing", JRRAS 7 ,April 2011.

[8] Garima Malik and Amandeep Singh Sappal, "Adaptive Equalization Algorithms:An Overview", (IJACSA) International Journal of Advanced Computer Science and Applications,Vol. 2, No.3, March 2011. 